\title{
Preparation And Bio Pharmaceutical Evaluation Of Chronopharmaceutical Drug Delivery System Of Metoprolol
}

\author{
Apollo James ${ }^{1}$, Mohanraj Palanisamy ${ }^{1,2}$, Jasmina Khanam ${ }^{1}$, Arun Radhakrishnan ${ }^{3}$ \\ ${ }^{1}$ Department of Pharmaceutical Technology, Jadavpur UniversitY, Kolkata 700 032, West Bengal, India \\ ${ }^{2}$ Department of Pharmaceutics, Nandha College of Pharmacy, Erode- 638 052, Tamilnadu, India \\ ${ }^{3}$ Department of Pharmaceutics, JSS college of Pharmacy, JSS Academy of Higher Education and Research, \\ Nilgris, Tamilnadu, India \\ E-Mail:ajamespharma@gmail.com
}

\begin{abstract}
The basic purpose of constructing drug delivery systems is to design when and where the drug will be released. The episode of many biological events is really important for such knowledge. Metoprolol pulsatile drug delivery system was developed for this purpose, which can release the drug when blood pressure needs to be modulated in the early morning. The Cup and core techniques were used to build this system, which included immediate release (IR), sustained-release (SR), and a polycaprolactone plug layer. The formulation of the ingredients was facilitated by various preformulation studies. The IR and SR tablets were bilayered, with polycaprolactone entirely coating the IR layer. The IR and SR tablet release profiles were optimised for the F5 batch, which was then used to construct a pulsatile drug delivery system. Clinical trials were conducted with the prepared tablet, which included the use of $\mathrm{BaSO} 4$ tagged tablets for X-ray examinations. All of the findings indicated the optimal drug release of metoprolol, which can be used for individuals who are more prone to blood pressure abnormalities in the morning.
\end{abstract}

Keywords: Chronopharmaceutial, Metoprolol, BaSO4 labelled, Pulsatile Drug Delivery

\section{INTRODUCTION}

Time is an important variable in drug development and delivery. Biological processes have been shown to have cycles of various scales, ranging from very brief (ultradian) rhythms to rhythms with a period of about one day (circadian) to rhythms with longer cycles, such as a week, a month, or even longer. Endogenous biological clocks, or time keeping systems, generate these rhythms rather than being a passive response to environmental changes. As a result, there is an abundance of data of chronopharmacotherapeutics, ranging from basic chronobiology to clinical applications (chronotherapy). (1).

The chronotherapeutical is a unique medication delivery system that synchronises drug availability with disease-related rhythms. If disease symptoms appear at a given time of day or night, regular dosage forms are inadequate to meet the needs of the situation. The substance in a controlled release medication is released at a steady rate rather than as and when needed. In recent years, the chronopharmaceutical drug delivery system has become increasingly important in the treatment of chronic diseases such as hypertension, allergic rhinitis, rheumatoid arthritis, nocturnal asthma, osteoarthritis, peptic ulcer, and cancer. (2). 
Pulsatile Drug Delivery System (PDDS) can be defined as the drug gets released after a particular lag time in relation to the circadian rhythm of the disease. Time-controlled pulsatile release (single or multiple unit system), internal stimuli induced release, and external stimuli induced pulsatile release systems are the three primary classes of PDDS based on the pulsed regulation of drug release. PDDS can also be divided into three categories based on the dosage form: capsules, pellets, and tablets. The 'core and cup' tablet is suitable for the chronotherapeutics system in tablet dosage form. A appropriate polymer/polymer coating controls the release of medication in flux at a specific time after a lag. (3).

Capillary resistance and vascular reactivity are higher in the morning and decrease later in the day in cardiovascular diseases; platelet aggregation is increased, resulting in blood hypercoagulability; as a result of this action, the risk of myocardial infarction and sudden cardiac death is higher in the early morning. Heart attacks are five to six times more likely to strike between the hours of 1 and 5 a.m., and studies show that morning heart attacks are more severe than those that occur later in the day. Typically, cardiovascular disorders begin with high blood pressure, which is a key risk factor for coronary heart disease and ischemic and hemorrhagic stroke, as well as peripheral vascular disease, renal impairment, retinal haemorrhage, and vision impairment. Raised blood pressure is probably responsible for 7.5 million fatalities worldwide, or 12.8 percent of all deaths (4).

Many hypertension patients, especially those at high risk for cardiovascular disease, are prescribed beta-blockers as first-line therapy. They're also used to treat various heart problems like congestive heart failure and post-myocardial infarction. $\beta$-blockers have a greater impact on diurnal blood pressure reduction, which matches well with the circadian pattern in sympathetic tone. Rather than giving patients the maximum tolerable dose of -blocker on a continuous basis, it may be preferable to timing the $\beta$-blockade to coincide with the highest levels of sympatho-excitation. By permitting periods of reduced $\beta$-blockade during lower-risk periods, this may lead to the increased exercise tolerance in persons with chronotropic incompetence (inadequate heart rate response) (5). Metoprolol is an antihypertensive cardioselective competitive beta-1 adrenergic receptor antagonist with no intrinsic sympathomimetic action. Metoprolol works by blocking beta 1adrenergic receptors in the myocardium, lowering the frequency and force of myocardial contraction and lowering cardiac output. This drug may also inhibit renin secretion, resulting in lower levels of angiotensin II, reducing vasoconstriction and aldosterone secretion (6). Metoprolol was classified as a 'Class I' drug in the Biopharmaceutics Classification System with high solubility and permeability.

The aim of the proposed work is to develop suitable 'Pulsatile dosage form' which falls under the category of Chronopharmaceutical drug delivery system'. Model drug chosen was Metoprolol with an objective of delivering drug during specific time of day or night and reducing the dosing frequency, minimizing adverse drug reaction and to improve patient compliance by enhancing the therapeutic efficacy of the drug.

\section{MATERIALS AND METHODS}

\subsection{MATERIALS}

The Pure Drug Metoprolol was purchased from Carbanio.com. Polymers and excipients such as Polycaprolactone, HPMC, Lactose, Sorbitol, Magnesium stearate and Talc was obtained from SD fine chemicals. Micro Crystalline Cellulose and Sodium Starch Glycolate were received from Lobachemie. 


\subsection{METHODS}

\subsubsection{Preformulation Studies}

Preformulation is the primary level of product development where the physiochemical properties of the active pharmaceutical ingredient and excipients which has the effect on the product formulation and performance can be studied (7). The preformulation studies are follows

\subsubsection{Analytical Method Development}

\section{Preparation of Calibration Curve}

$50 \mathrm{mg}$ of Metoprolol was dissolved in $50 \mathrm{ml}$ of methanol [stock-I $(1000 \mu \mathrm{g} / \mathrm{ml})$ ]. $1 \mathrm{ml}$ was taken from the above solution and made up with $10 \mathrm{ml}$ of methanol [stock-II $(100 \mu \mathrm{g} / \mathrm{ml})$ ]. Using $0.2 \mathrm{M}$ Phosphate buffer ( $\mathrm{pH}$ 6.8), the above stock-II solution was diluted, to obtain series of dilutions containing 2, 4, 6,8,10 $\mu \mathrm{g} / \mathrm{ml}$ of solution. The absorbance of the above dilutions was measured at 220nm using UVSpectrophotometer taking 0.2M phosphate buffer (pH 6.8) as blank. Then a graph was plotted by taking Concentration on X-Axis and Absorbance on Y-Axis which gives a straight line Linearity of standard curve, which was assessed from the square of correlation coefficient $\left(\mathrm{R}^{2}\right)$.

\subsubsection{Flowability Studies}

\section{Angle of repose}

It is defined as, the maximum angle possible between the surface of the pile of the powder and the horizontal plane. Angle of repose measures the frictional force in a loose powder, in which fixed funnel method was used to measure the angle of repose.A funnel was fixed at a given height, above the graph sheet, which was placed on a flat horizontal surface. The blend was carefully pored through the funnel until the apex of the conical pile just touches the tip of the funnel. The radius (r) of the base of the conical pile was measured (8). The angle of repose was calculated using the following formula:

$\theta=\operatorname{Tan}^{-1}(\mathrm{~h} / \mathrm{r})$

Where,

$\mathrm{h}=$ Height of the cone

$r=$ Radius of the cone base

\section{Bulk density}

Bulk density, is defined as the mass of the powder divided by the bulk volume and is expressed as $\mathrm{gm} / \mathrm{cm} 3$. Particle size distribution, Particle shape and adherence of particles determine bulk density of powders. $10 \mathrm{gm}$ powder blend was sieved and introduced into measuring cylinder, without compacting (9). Bulk density was calculated using,

Bulk Density $=\mathrm{M} / \mathrm{V}_{\mathrm{o}}$

Where, 
$\mathrm{M}=$ weight of sample

$\mathrm{V}_{\mathrm{o}}=$ apparent volume of powder

\section{Tapped density}

Tapped density is defined as the mass of the powder divided by the bulk volume after the mechanically tapping. $10 \mathrm{~g}$ of powder blend was placed in measuring cylinder and tapped for 100 times using mechanical tapped density tester (10). Tapped density was calculated using,

Tapped density $=\mathrm{M} / \mathrm{V}$

Where,

$\mathrm{M}=$ Weight of sample

$\mathrm{V}=$ Tapped volume of powder

\section{Measures of powder compressibility}

The Compressibility Index (Carr's Index) is a measure of the propensity of a powder to be compressed, which is determined using bulk and tapped densities. Inter particulate interactions are generally less significant in free flowing powders and bulk and tapped densities will be closer in value.

For poorer flowing materials, there are frequently greater interparticle interactions, and a greater difference between the bulk and tapped densities will be observed (11). These differences are reflected in the Compressibility Index which is calculated using the following formulas:

Carr's Index $=[($ tap $-b) /$ tap $] \times 100$

Where,

$\mathrm{b}=$ Bulk Density

Tap $=$ Tapped Density

\section{Hausner's Ratio}

Hausner's ratio expresses the flowability of the powders or granules (12). Hausner ratio was calculated by using the following equation:

Hausner Ratio $=\mathrm{V}_{0} / \mathrm{V}_{\mathrm{f}}$

where, $\mathrm{V}_{\mathrm{o}}=$ unsettled apparent volume $\left(\mathrm{cm}^{3}\right)$,

$\mathrm{V}_{\mathrm{f}}=$ final tapped volume $\left(\mathrm{cm}^{3}\right)$

\subsubsection{Drug - excipient Compatibility Studies}

\section{Differential scanning Calorimetry}

The thermograms of drug and excipients were recorded on model differential scanning calorimeter. An empty pan was used as a standard. About 1 gram of sample including pure drug (metoprolol), physical mixture (drug, excipients) were separately weighed and sealed in a small aluminium pan and 
it was heated up to $20-400^{\circ} \mathrm{C}$ at the heating rate of $10^{\circ} \mathrm{C} / \mathrm{min}$ with constant purging of dry nitrogen $50 \mathrm{ml} / \mathrm{min}$.For the reference, a blank pan was sealed and used. Using automatic thermal analyzer system, DSC thermogram was obtained. The DSC thermo gram obtained from this study was used for determining the compatibility between the drug and the excipients.

\subsubsection{Solubility Studies}

As per BCS guidelines, saturation solubility studies of MetaprololSuccinate was conducted. 25mg of Metoprolol Succinate was added to $250 \mathrm{ml}$ of each medium $(0.1 \mathrm{~N} \mathrm{HCl} \mathrm{pH} \mathrm{1.2,} \mathrm{Acetate} \mathrm{Buffer} \mathrm{pH} \mathrm{4.5,}$ Distilled water $\mathrm{pH} 7.0$ and Phosphate buffer $\mathrm{pH}$ 6.8) placed in 500ml Conical flask.

\subsubsection{Melting Point Determination}

Melting point determination helps in specifying the solid crystalline materials by thermal analysis. The melting point can be determined by using the melting point apparatus.

\subsubsection{PREPARATION OF PULSATILE METOPROLOL TABLETS}

\subsubsection{Preparation of metoprolol core tablet}

Table 2 shows the ingredients of a metoprolol tablet. Separately, all of the ingredients were sieved with no. 45. The geometric addition approach was used to combine metoprolol succinate, microcrystalline cellulose, sorbitol, and talc. The Core in Cup tablet was made as a bilayered tablet, with a prolonged release layer as the first layer and an immediate release layer with direct compression as the second layer. It was designed with the required amount of ingredients for the immediate and sustained release layers. An $8 \mathrm{~mm}$ punch was used to punch the bilayered tablet.

To make a bilayered tablet, the immediate release layer was punched first with a low compression force, and then the sustained release layer was inserted and punched above it. Various batches of immediate and sustained release tablets have been developed in order to obtain an optimal drug release profile.

Table 1: Composition of Metoprolol Core Tablet

\begin{tabular}{|c|c|c|c|c|c|c|}
\hline Ingredients & F1 & F2 & F3 & F4 & F5 & F6 \\
\hline \multicolumn{7}{|c|}{ Immediate Release Tablet } \\
\hline Metoprolol & 14 & 14 & 14 & 14 & 14 & 14 \\
\hline $\begin{array}{c}\text { Cross carmellose } \\
\text { sodium }\end{array}$ & - & - & - & 3 & 6 & 9 \\
\hline $\begin{array}{c}\text { Sodium Starch } \\
\text { Glycolate }\end{array}$ & 3 & 6 & 9 & - & - & - \\
\hline MCC & 10 & 10 & 10 & 10 & 10 & 10 \\
\hline Sorbitol & 37.1 & 33.85 & 30.6 & 37.1 & 33.85 & 30.6 \\
\hline
\end{tabular}




\begin{tabular}{|c|c|c|c|c|c|c|}
\hline Talc & 0.325 & 0.325 & 0.325 & 0.325 & 0.325 & 0.325 \\
\hline $\begin{array}{c}\text { Magnesium } \\
\text { Stearate }\end{array}$ & 0.325 & 0.325 & 0.325 & 0.325 & 0.325 & 0.325 \\
\hline \multicolumn{7}{|c|}{ Sustain Release Polymeric Layer } \\
\hline Metoprolol & 12 & 12 & 12 & 12 & 12 & 12 \\
\hline HPMC & 10 & 15 & 20 & 10 & 15 & 20 \\
\hline $\begin{array}{l}\text { PVP in alcoholic } \\
\text { solution }\end{array}$ & $2.8 \%$ & $2.8 \%$ & $2.8 \%$ & - & - & - \\
\hline $\mathrm{MCC}$ & 60 & 55 & & 60 & 57 & 50 \\
\hline Lactose & 22 & 22 & 22 & 22 & 22 & 22 \\
\hline Talc & 0.5 & 0.5 & 0.5 & 0.5 & 0.5 & 0.5 \\
\hline $\begin{array}{c}\text { Magnesium } \\
\text { Stearate }\end{array}$ & 0.5 & 0.5 & 0.5 & 0.5 & 0.5 & 0.5 \\
\hline \multicolumn{7}{|c|}{ Coating } \\
\hline Polycaprolatone & QS & QS & QS & QS & QS & QS \\
\hline
\end{tabular}

\subsubsection{Coating of core in cup tablet}

The drug was coated on a bilayer tablet to achieve pulsatile release. Polycaprolactone (hydrophobic polymer) was dissolved in chloroform to generate a viscous solution for the coating. This solution was used to spray coat the immediate release layer of a bilayered tablet on all sides, leaving the sustained release layer untreated.

\subsubsection{EVALUATION OF PREPARED CORE TABLETS}

\subsubsection{Disintegration test}

For tablets, the first important step towards drug dissolution is breakdown of the tablets into granules or primary powder particles, a process known as disintegration. All USP tablets must pass a test for disintegration, which is conducted in vitro using a disintegration test apparatus. The apparatus consists of a basket-rack assembly containing six open-ended transparent tubes of USP-specified dimensions, held vertically upon a 10-mesh stainless steel wire screen. During testing, a tablet is placed in each of the six tubes of the basket, and through the use of a mechanical device, the basket is raised and lowered in a bath of fluid (e.g. water, or as prescribed in the individual drug monograph) at 29 to 32 cycles per minute, the wire screen always below the level of the fluid.

\subsubsection{Hardness test:-}


Monsanto hardness tester was employed for this test. Force required to break the tablet is determined in this test. The tablet was placed between two pieces. The strength necessary to break the tablet was considered. The crushing strength test was executed on 8 tablets from the formulation.

\subsubsection{Weight Variation Test}

20 tablets were weighed individually and average weight was calculated. The tablet pass the U.S.P. test if no more that 2 tablets are outside the percentage limit and if no tablet differs by more than 2 times the percentage limit. The weight variation was calculated and results were reported.

Average weight $(\mathrm{mg})=$ Total Weight $/ 20$

\subsubsection{Friability test}

It is an important parameter to know whether the drug undergoes any capping, lamination etc during shifting of the products. Friability test helps in identifying the robustness of the tablets. The friability can be determined by

Friability $(\%)=\frac{\text { Initial weight-finnal weight }}{\text { Inîtial weight }} \times 100$

\subsubsection{In vitro drug release studies}

The core tablets were tested in vitro using a USP dissolution apparatus II at $37^{\circ} \mathrm{C}$ with $900 \mathrm{ml}$ of phosphate buffer $(\mathrm{pH}$ 6.8) and a stirring rate of 50 revolutions per minute. The USP Dissolution device II was used to test in vitro drug release for immediate release, sustained release, and bilayered tablets (Paddle). The experiment was carried out with $900 \mathrm{ml}$ of PBS 6.8 and a $37^{\circ} \mathrm{C}$ temperature. The dissolution medium and the paddle were set up with the formulation. The shaft was fixed and rotated at 50 revolutions per minute. At various time intervals, $1 \mathrm{ml}$ of sample was extracted from the basket. Following the removal of the sample, the same amount of PBS was replaced with new PBS. The filtered sample was measured with UV absorbance at $220 \mathrm{~nm}$. The following calculation was used to compute the cumulative percentage drug release.

Concentration $(\mu \mathrm{g} / \mathrm{ml})=$ Absorbance \pm intercept $\times$ slope

Amount release $(\mathrm{mg})=$ Concentration $\times$ Bath Volume $\times$ Dilution factor $/ 1000$

Percentage drug release $=$ Amount released $\times$ label claim

Cumulative release $(\%)=$ volume withdraw $\times$ PDR $(\mathrm{t}-1)+$ PDR Bath volume

Whereas,

$\mathrm{PDR}=$ percentage drug release at time $\mathrm{t}$

PDR $(\mathrm{T}-1)=$ previous percentage drug release at time $\mathrm{t}$

\subsubsection{Modeling of release profiles}

The release data was treated according to Higuchi, korsmeyer-peppas, Hixson-crowell, and weibll models, as well as zero and first order patterns using add in DD solver software to establish Metoprolol release kinetics for the specified formulas.

\subsubsection{Water uptake and erosion studies}

Type 1 dissolution apparatus was used for the water uptake and erosion studies. Prior to the experiment, tablets and empty baskets were weighed. Individual tablets were withdrawn at regular 
time intervals. To remove the excess liquid, basket and tablet were blotted and then reweighed. At $80^{\circ} \mathrm{C}$, wetted tablets were dried until a constant weight is reached. Once after drying, tablets were allowed to cool in a desiccator and then reweighed. The experiment was done in duplicate. The extent of erosion, $\mathrm{E}(\%)$, was determined as follows:

$\mathrm{E} \%=100 \times\left(\mathrm{W}_{\mathrm{i}}-\mathrm{W}_{\mathrm{f}}\right) / \mathrm{W}_{\mathrm{i}}$

Where,

$\mathrm{W}_{\mathrm{i}}$ - initial starting dry weight

$\mathrm{W}_{\mathrm{f}}$ - final dry weight of the same dried and partially eroded tablet

The increase in weight (uptake) due to absorbed water A (\%), was calculated at each time point using:

$\mathrm{A} \%=100 \times\left(\mathrm{W}_{\mathrm{w}^{-}}-\mathrm{W}_{\mathrm{f}}\right) / \mathrm{W}_{\mathrm{f}}$,

Where,

$\mathrm{W}_{\mathrm{w}}$ - Weight of the wet tablet before drying.

$\mathrm{W}_{\mathrm{f}}$ - Final dry weight of the same dried and partially eroded tablet.

\subsubsection{Stability studies}

Under both standard long-term and accelerated storage conditions, the selected formulations were assessed for stability according to the International Council of Harmonization (ICH) standards. Petri dishes made of polypropylene were used to hold the tablets. Half of the tablets were placed in a desiccator with anhydrous calcium chloride and stored at $25^{\circ} \mathrm{C}$ and $60 \%$ relative humidity for three months. For three months, the other half was stored in a stability cabinet (Climacell-MMM, Germany) set at $40^{\circ} \mathrm{C}$ and $75 \%$ relative humidity. The tablets were tested for physical appearance and in vitro drug release at predetermined intervals.

\subsubsection{Floating tests}

An in-vitro experiment was carried out to estimate the floating time. The buoyancy of tablet formulations was examined in a $100 \mathrm{ml}$ beaker containing $80 \mathrm{ml} 0.1 \mathrm{M}$ Hydrochloric acid. The floating time, or the point at which the tablet of each formula begins to sink, was recorded. Each experiment was repeated three times. The image of the tablets was captured using a digital camera in each case.

\subsubsection{Verification of tablets buoyancy in healthy volunteers:}

After obtaining informed written consent, two healthy male volunteers took part in the study. The participants were 30 and 34 years old, with heights of 157 and $164 \mathrm{~cm}$ and weights of 57 and $63 \mathrm{~kg}$ respectively. The Nandha Medical College and Hospital Protection of Human Subjects Committee approved the study, and the methodology follows the hospital's ethical committee's requirements.

A full medical history, physical examination, and haematological and biochemical laboratory investigations were used to establish the health state of the volunteers. The participants were told not to take any medications for a week before and throughout the trial.

All other ingredients were preserved in the same quantities and one of the selected formulations was made X-ray opaque by replacing $30 \mathrm{mg}$ of Sorbitol with barium sulphate (barium sulphate labelled tablets). This amount was discovered through experimentation to allow X-ray opacity without compromising tablet buoyancy. The alternate version, which has $40 \mathrm{mg}$ barium sulphate in the cup, was X-ray opaque by default. 
The volunteers were offered a low-calorie meal after fasting for the night. One F Tablet was given to one individual and one barium sulphate labelled F tablet was given to the second subject with $200 \mathrm{ml}$ of water half an hour later.

Before and after drug delivery, the volunteers were subjected to abdomen X-ray imaging in a standing position. For all photos, the distance between the X-ray source and the subject was kept constant.

\subsubsection{Statistical analysis}

The data is presented as a mean standard deviation. The student's t-test was used for all statistical comparisons, and a p-value of 0.05 was considered significant.

\section{RESULTS AND DISCUSSION}

\subsection{Flowability studies}

It is necessary to have good flow and compacting qualities for direct compression of materials. Angle of repose of $31-35^{\circ}$, according to USP, indicates good flow quality. Fair flow is indicated by a Hausner ratio of less than 1.25 and a vehicles index of 16-20.

Metoprolol express the angle of repose of $20.22 \pm 0.24-27.12 \pm 0.22$, showing excellent flow properties. Table 3 depicts the pre-compression parameters for Immediate Release Layer.

Table 2: Pre-compression Parameters for Immediate Release Layer

\begin{tabular}{|l|l|l|l|l|l|}
\hline Batch & $\begin{array}{l}\text { Angle of } \\
\text { Repose } \\
\left({ }^{\circ}\right.\end{array}$ & $\begin{array}{l}\text { Bulk } \\
\text { density } \\
(\mathbf{g} / \mathbf{c c})\end{array}$ & $\begin{array}{l}\text { Tapped } \\
\text { density } \\
(\mathbf{g} / \mathbf{c c})\end{array}$ & $\begin{array}{l}\text { Carr's } \\
\text { Index } \\
(\%)\end{array}$ & $\begin{array}{l}\text { Hausner's } \\
\text { Ratio }\end{array}$ \\
\hline F1 & 26.8 & 0.25 & 0.29 & 13.79 & 1.16 \\
\hline F2 & 25.6 & 0.25 & 0.28 & 10.71 & 1.12 \\
\hline F3 & 29.8 & 0.27 & 0.31 & 12.90 & 1.14 \\
\hline F4 & 25.1 & 0.26 & 0.30 & 13.33 & 1.15 \\
\hline F5 & 27.8 & 0.31 & 0.35 & 12.90 & 1.12 \\
\hline F6 & 26.2 & 0.25 & 0.28 & 10.71 & 1.12 \\
\hline F7 & 29.1 & 0.30 & 0.34 & 11.76 & 1.13 \\
\hline F8 & 28.5 & 0.26 & 0.29 & 10.34 & 1.11 \\
\hline
\end{tabular}

Carr's index and Hausner ratio of metoprolol formulations were 10.34 to 13.79 and 1.11 to 1.16 respectively. This showed good flow properties of the formulation blend.

\subsection{DSC Studies}

The DSC investigations were carried out to see if metoprolol and excipients were compatible. The resulting thermogram clearly demonstrated that metoprolol and the excipients don't have any physical contact. 


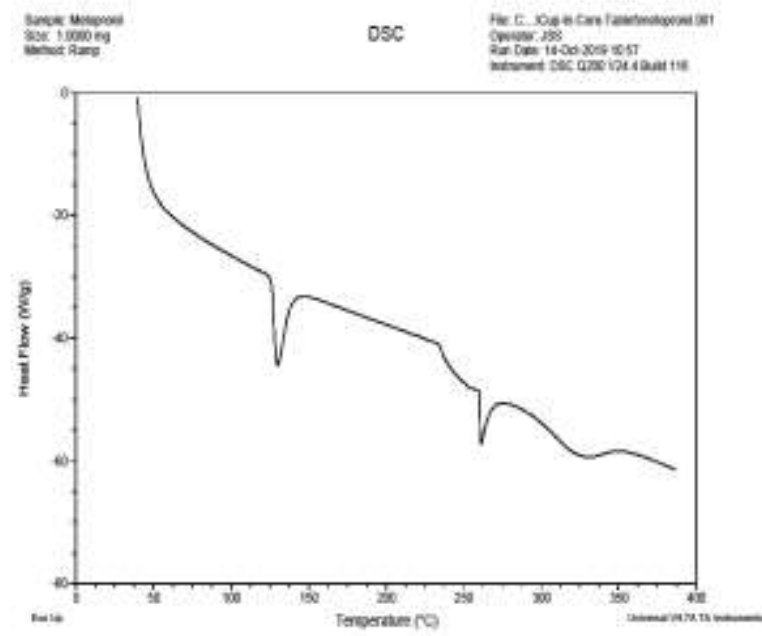

Fig.no.3.2.1: Metoprolol

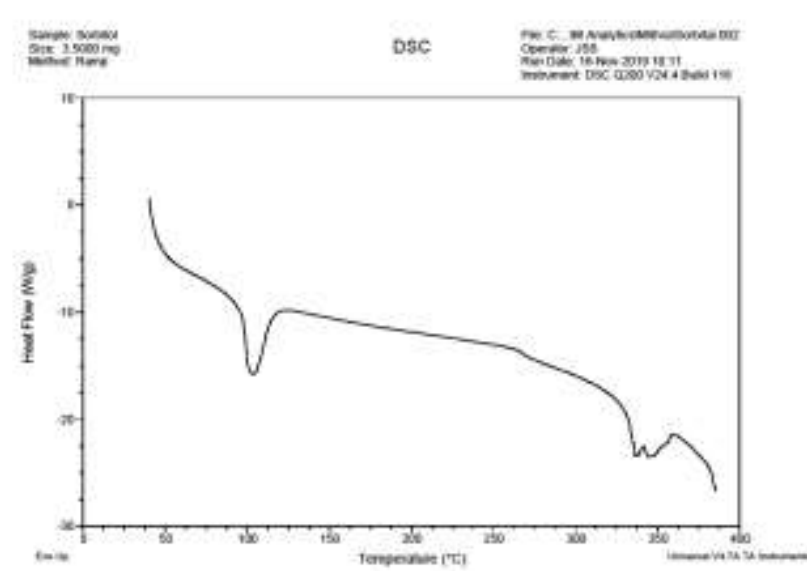

Fig.no.3.2.3: Sorbitol

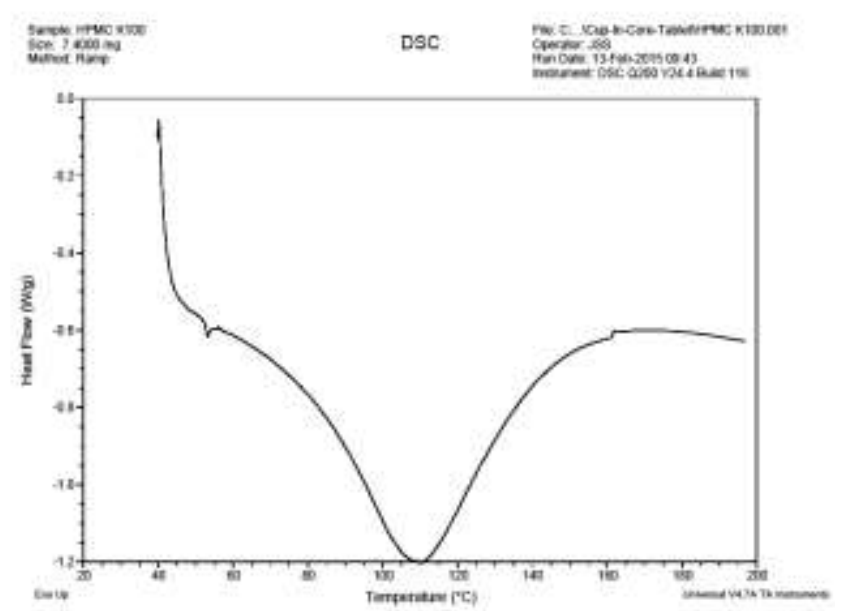

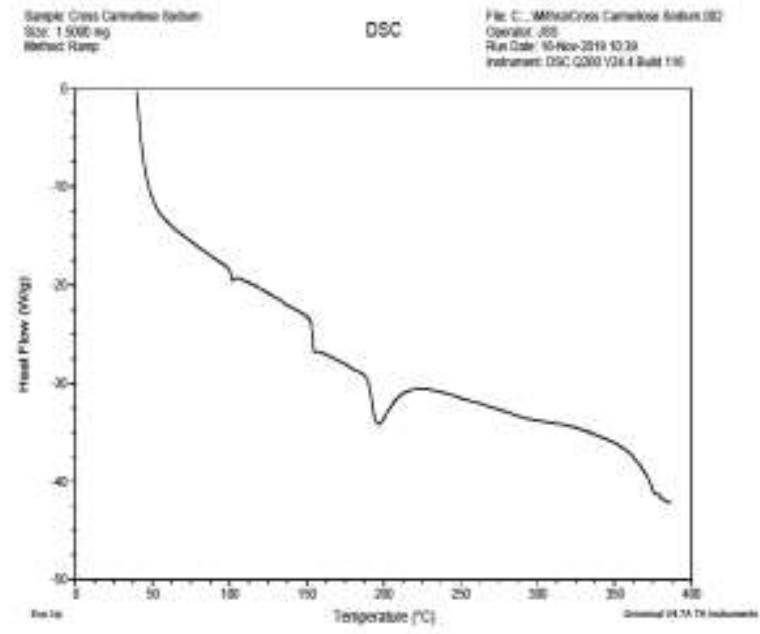

Fig.no.3.2.2: Cross carmellose

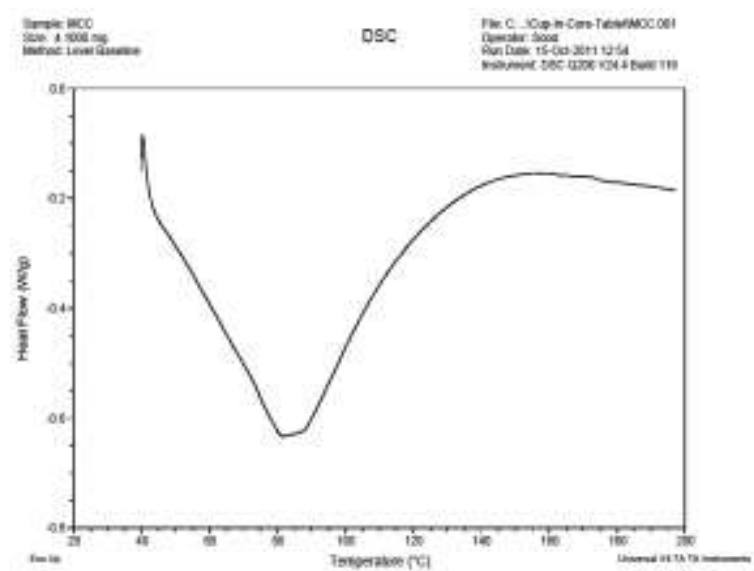

Fig.no3.2.4 :MCC

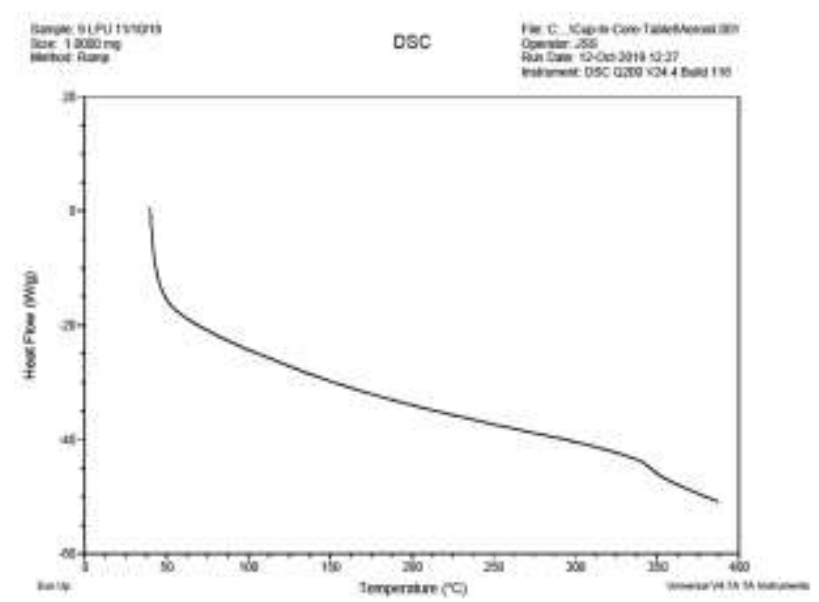


Fig. no.3.2.5: HPMC

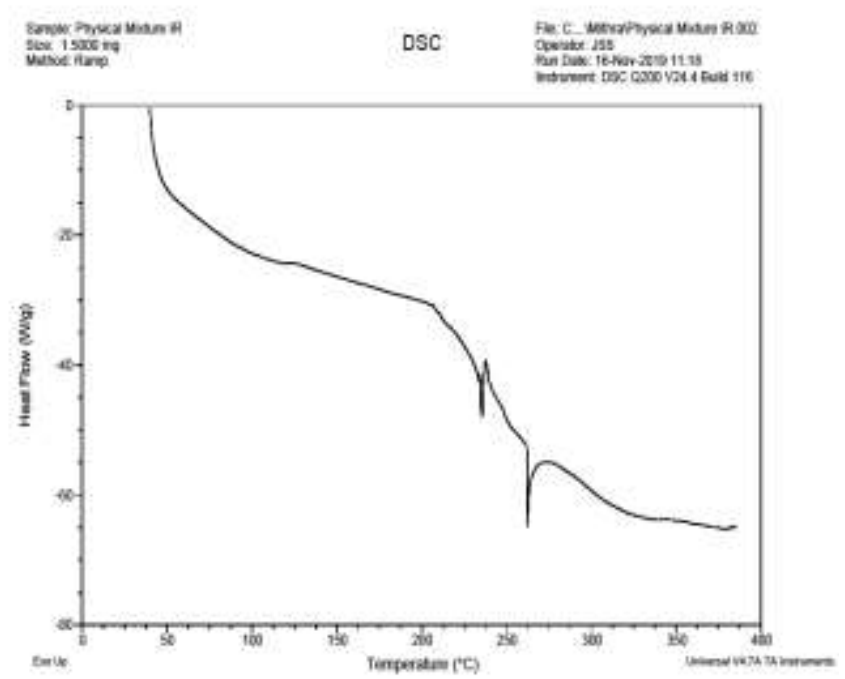

Fig.no.3.2.7: PM IR
Fig. no.3.2.6: Magnesium Stearate

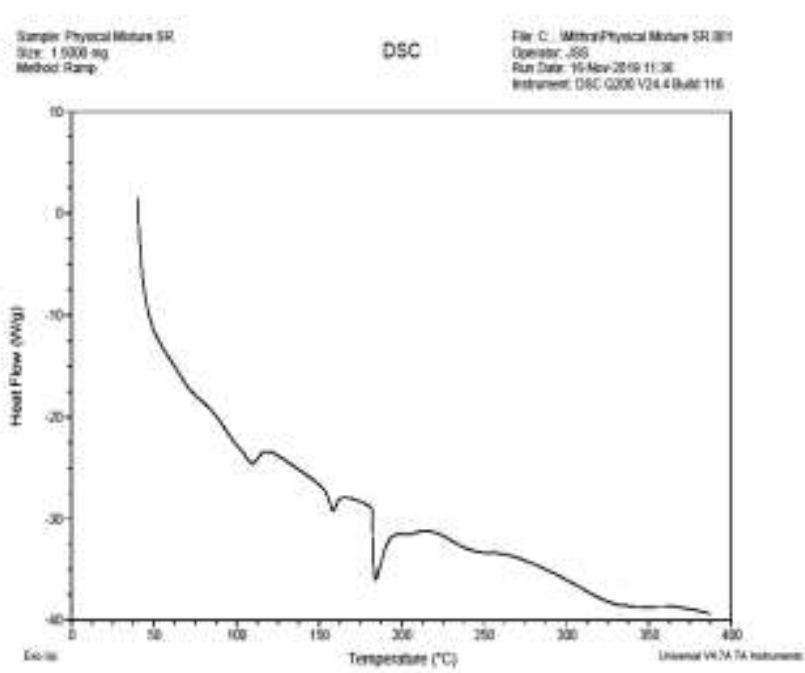

Fig. no.3.2.8: PM SR

\subsection{Solubility study}

In $0.1 \mathrm{~N} \mathrm{HCl}$, phosphate buffer ( $\mathrm{pH} 6.8$ ), and distilled water, metoprolol solubility was $0.067,42.7$, and $0.18 \mathrm{~g} / \mathrm{L}$, respectively. These solubility values are associated with metoprolol's mildly acidic nature. In acidic media, it has low solubility, but in alkaline media, it has high solubility.

\subsection{Evaluation of the prepared core tablet}

All of the tests for core tablets that were conducted were successful. The thickness $(3.48 \pm 0.04 \mathrm{~mm})$ and diameter $(6.07 \pm 0.01 \mathrm{~mm})$ of the tablets were also consistent. The hardness of the material was determined to be $5.0 \pm 0.3 \mathrm{~kg} / \mathrm{cm} 2$. The percentage weight loss was 0.19 percent in terms of friability. The weight of 20 tablets was $164 \pm 0.9 \mathrm{mg}$ on average. In all medium, the disintegration time of the core tablet formulation was instantaneous, but the disintegration period of the F5 formulation was less than 1 minute. As a result, it was determined that cup compression has no effect on the disintegration time of the core tablet.

\subsection{Assay method validation}

The linearity, inter-day precision, and intra-day precision data were used to validate the spectrophotometric assay's parameters.

Linearity:

Metoprolol showed a linear relationship in $0.1 \mathrm{~N} \mathrm{HCl} \& \mathrm{pH} 6.8$ over a concentration range of (1-3 $\mathrm{mg} \%)$ in both media with a value of coefficient of determination $\left(\mathrm{R}^{2}\right) 0.998 \& 0.9995$ respectively.

\section{PRECISION:}

i) Inter-day Precision:

In $0.1 \mathrm{M} \mathrm{HCl}, \% \mathrm{CV}$ is not more than $5.13 \%$, 
In $\mathrm{pH} 6.8, \% \mathrm{CV}$ is not more than $6.44 \%$.

ii)

Intra-Day Precision:

In $0.1 \mathrm{M} \mathrm{HCl}, \%$ is not more than $2.49 \%$,

In $\mathrm{pH} 6.8, \mathrm{CV}$ is not more than $1.81 \%$.

No interference was reported from the other components of the tablets under the selected $\pi_{\max }$ in both media.

\subsection{In-vitro drug release studies}

In vitro release profile of metoprolol from the core tablet in 100mL.buffer with $\mathrm{pH} 6.8$ for 3 hours at $37 \pm 0.5 \AA$ and 50rpm was conducted to investigate the release profile of metoprolol from the core tablet in a medium.

The release of metoprolol from the core tablet was compared from a core-in-cup formulation without the plug layer to see how the cup affected drug release. The difference in metoprolol release between the $\mathrm{C}$ and bilayer tablets was not statistically significant $(\mathrm{P}>0.05)$. As a result, the EC cup has no effect on metoprolol release.

The plug layer, which is made up of polycaprolactone, absorbs water when the core-in-cup tablet comes into contact with the medium which leads to the polymer stretches and swells. With time, the plug swells and expands, providing a barrier that prevents liquids from coming into contact with the core tablet's surface. The type of polymer employed can have an influence on this technique. It's also possible that the plug layer's enlargement will destabilize the plug, causing it to erode or be removed over time. As a result, the rate at which the plug layer polymer erodes is controlled by the plug polymer's swelling and instability. Finally, the plug is totally eroded and water entry into the core increases dramatically, resulting in rapid medication release, depending on the properties of each polymer. Moreover, in the same formulation strategies, to provide a quick release following the lag period, $\mathrm{CCNa}$ was added as a superdisintegrant to the core formulation. $\mathrm{CCNa}$ reduces the time to disintegrate and improves the solubility of poorly soluble medicines. Priority and severe swelling are the two disintegration mechanisms linked with $\mathrm{CCNa}$, with the latter being the most critical. When the percentage of $\mathrm{CCNa}$ does not reach the point where disintegration time is the least $(7.6 \%)$, the swelling process takes over.

As will be demonstrated, the analysis of water uptake-erosion in the Polycaprolactone plug layer can fairly explain the behavior of the top plug layer. With the passage of time, the Polycaprolactone layer swelled and expanded rapidly, reaching its maximum water uptake (121.7\%) after 2 hours. When the medium $\mathrm{pH}$ was changed from 1.2 to 6.8 , there was a significant drop in water intake, as well as significant erosion. Polycaprolactone erosion accelerated from $10.4 \pm 2.2$ percent after 2 hours to 27.2 \pm 1.9 percent after 2.5 hours. As a result, Polycaprolactone had a faster release after the lag time. It is clear that our findings are consistent with those of Efentakis et al.

The utilized HPMC grade, S Methocel®K4M, was distinguished by its viscosity, which was $4000 \mathrm{cp}$. The gel layer was sufficiently resistant to widespread erosion even after hydration, which was followed by an increase in route length. As a result, drug release from the core-in-cup tablet technology does not match the pulsatile release rate profile.

After adjusting the $\mathrm{pH}$ from 1.2 to 6.8 , the in vitro release rate of metoprolol was found to be the most convenient among previously examined tablet formulations, with an optimal lag period followed by rapid pulsatile drug release. 
The marketed tablet had no lag time and 25 percent drug release after 2 hours followed by entire drug release after 3.5 hours. As a result, the core-in-cup system's delayed impact is clarified.

All of the generated core-in-cup tablets were floating due to the low density of the EC utilized (0.4 $\mathrm{g} / \mathrm{cm} 3)$. The suppression of floating of the F5 tablet formulation was necessary because these tablets had a long floating time ( 3 hours \& 40 minutes, as evidenced by the results of the in vitro floating time measurement), which resulted in an increase in gastric retention and a delay in the arrival of the tablets to the absorption window. \% metoprolol release from F5 in $0.1 \mathrm{MHCl}$ was about 17 percent during this floating duration (the drug release exceeded 10 percent $\&$ the lag time has ended before leaving the stomach). As a result, F5 tablets should be allowed to sink for 2 hours (lag time) before leaving the stomach.

Preliminary testing was conducted to determine the best high density material to be integrated into the EC cup to prevent pill drifting. $\mathrm{ZnO}$ and $\mathrm{BASo} 4$, which have densities greater than that of stomach contents $(1.004 \mathrm{~g} / \mathrm{cm} 3)$, were used in 1:2, 1:2.75, 1:3, and 1:4 ratios to EC powder in the studies. The lowest possible ratio of 1:2.75 was chosen and integrated with the EC cup of f2 to generate an incore-cup single pulse tablet formulation (f5), whose composition was displayed, thus limiting stomach retention of the tablets and hence accelerating the pulsatile release of metoprolo at $6.8 \mathrm{pH}$. After a 2-hour lag period in $0.1 \mathrm{M} \mathrm{HCl}, \mathrm{F} 5$ tablets released metoprolol quickly.

When a tablet is swallowed, it instantly sinks into the stomach's pylorus until gastric emptying (after 2-3 hours), as evidenced by X-ray radiography. No drug is released as long as the tablet is inside the stomach. Polycaprolactone rapidly erodes and metoprolol is released once the tablet moves from the stomach acidity to the higher $\mathrm{pH}$ of the duodenum (6.8), the metoprolol window of absorption.

To achieve good patient compliance and less frequent dosage ingestion, several multi-layered tablet designs are used to obtain two or more drug release pulses.

Metoprolol is released in two pulses by F5 tablet. The tablet floats in $0.1 \mathrm{M} \mathrm{HCl}$, and the first pulse is released immediately. The tablet is swept into the $\mathrm{pH} 6.8$ (corresponding to the metoprolol absorption window) as soon as it descends, allowing the second pulse to be released.

To allow for an appropriate lag time between the two consecutive pulses, F5 must be floated. It's also crucial to avoid as early release of the second pulse, which could circumvent the duodenum's absorption window, lowering metoprolol bioavailability.

Floating lag time and duration of floating of F5 tablets were $76 \pm 11 \mathrm{~s}$ and $162 \pm 17 \mathrm{~min}$ respectively. Finally, F5 is the met's chosen single pulse core-in-cup tablet information. After a lag time equal to the time it takes for the stomach to empty, the drug's pulse is released ( 2 hours). Meanwhile, metoprolol is released by F5 in two consecutive pulses with a fair lag time in between because of good floating property.

\subsection{Modeling of release profiles}

The value of R2-0.8503 indicated that metoprolol was released into the Korsmeyer-Peppas model. The " $n$ " exponent value was employed by Peppas in 1985 to characterise the various release processes. The associated release mechanism is quasi-Fickain diffusion with partly chain relaxation regulated process, since the $\mathrm{n}$ value for $\mathrm{F} 5$ formulation is 0.0887 .

Metoprolol's release fits into the Weibull model. The shape of the curve becomes sigmoidal with a turning point as $b$, the shape parameter, is $>1$;(3.679). Complex release is indicated by the sigmoidal curve form. A mechanism, since the rate of release does not alter in a predictable manner. In fact, the release rate rises nonlinearly until it reaches the inflection point, then falls asymptotically. 
Table 3: In-vitro Drug Release of Immediate Drug Release

\begin{tabular}{lllllll}
\hline \multirow{2}{*}{$\begin{array}{l}\text { Time } \\
\text { (hrs) }\end{array}$} & F1 & F2 & F3 & F4 & F5 & F6 \\
\cline { 2 - 7 } $\mathbf{1}$ & 22.35 & 25.36 & 19.5 & 0.23 & 0.82 & 0.37 \\
\hline $\mathbf{2}$ & 38.12 & 45.25 & 35.69 & 6.98 & 4.95 & 10.26 \\
\hline $\mathbf{3}$ & 49.5 & 56.27 & 48.67 & 35.64 & 42.15 & 43.98 \\
\hline $\mathbf{4}$ & 67.58 & 69.15 & 71.25 & 45.32 & 54.59 & 53.27 \\
\hline $\mathbf{5}$ & 89.92 & 95.64 & 85.63 & 76.25 & 81.51 & 79.61 \\
\hline $\mathbf{6}$ & 95.25 & 97.46 & 94.35 & 89.32 & 98.13 & 95.62 \\
\hline
\end{tabular}

Table 4: In-vitro Drug Release of Sustain Drug Release

\begin{tabular}{lllllll}
\hline $\begin{array}{l}\text { Time } \\
\text { (hrs) }\end{array}$ & F1 & F2 & F3 & F4 & F5 & F6 \\
\cline { 2 - 7 } $\mathbf{1}$ & 2.63 & 1.35 & 1.23 & 0.82 & 0.99 & 1.23 \\
\hline $\mathbf{2}$ & 10.12 & 6.54 & 9.64 & 4.95 & 5.32 & 3.21 \\
\hline $\mathbf{3}$ & 60.14 & 66.21 & 61.36 & 65.15 & 63.68 & 68.67 \\
\hline $\mathbf{4}$ & 65.62 & 71.14 & 70.19 & 75.59 & 75.12 & 71.32 \\
\hline $\mathbf{5}$ & 75.51 & 79.68 & 75.12 & 81.51 & 86.21 & 89.21 \\
\hline $\mathbf{6}$ & 89.23 & 91.26 & 86.61 & 98.13 & 96.51 & 95.32 \\
\hline
\end{tabular}

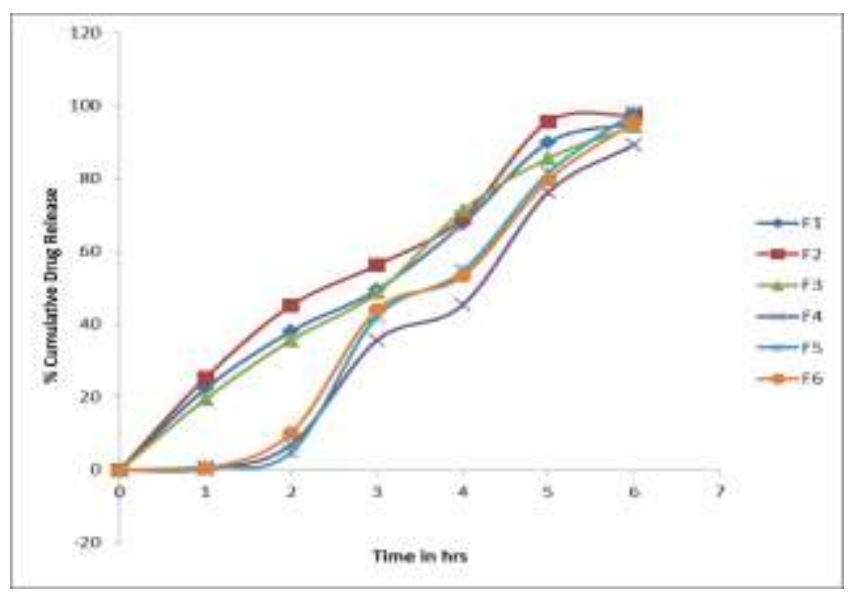

Figure. Release profile of Immediate Drug Release 


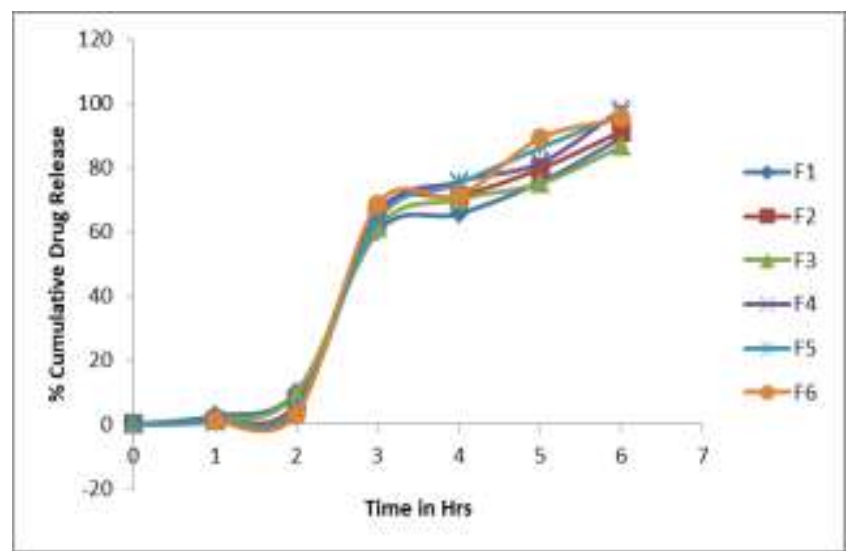

Figure. In-vitro Drug Release of Sustain Drug Release

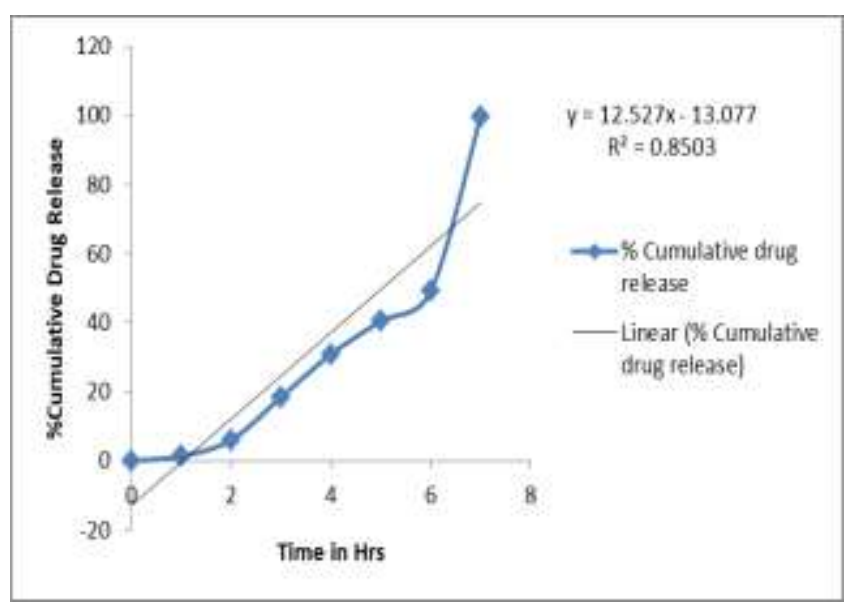

Figure 3: Invitro Drug Release of Bilayered Tablet

\subsection{Water uptake and erosion studies}

Since the Polycaprolactone plugged core-in-cup tablets of metoprolol showed the fastest pulsatile drug release after a lag time, a study of water uptake and Polycaprolactone was conducted. The amount of water absorbed and the amount of erosion of the Polycaprolactone layer are reflected in lag time value. When Polycaprolactone polymer is exposed to water, it swells and forms a viscous gel at the top layer surface, according to thorough observation. The maximum swelling was obtained during the trials, followed by disintegration of the polymeric layer.

When the $\mathrm{pH}$ of the medium was changed from 1.2 to 6.8 , the Polycaprolactone layer showed a rapid liquid uptake with a maximum between 1.5 and 2 hours, followed by a rapid decline owing to erosion. After 3.5 hours, the erosion was complete (31 percent erosion, calculated as a percentage of the initial core-in-cup tablet weight). The inflated layer disintegrated quickly after 2 hours due to the low viscosity of Polycaprolactone.

\subsection{Stability studies}

F5 and F6 tablets were stored at 25 degrees Celsius and 60\% relative humidity for 3 months (normal long-term storage settings) and 40 degrees Celsius and $75 \%$ relative humidity for 3 months (accelerated storage condition). After storage under both regular and accelerated settings, the rate of valsartan release from F5 did not alter significantly $(\mathrm{P}>0.05)$. 
Furthermore, during the study period, F5 and F6 did not demonstrate any significant changes in appearance. Under accelerated storage circumstances, there was a small plug layer swelling in both formulations at the conclusion of the study period, but it had no effect on the drug release profile $(\mathrm{P}>$ $0.05)$.

\subsection{Verification of tablets buoyancy in healthy volunteers}

Figure 8 shows radiographic images taken at various times after oral delivery of one tablet of each of the F5 and barium sulphate labelled B6 tablets. The high density $\mathrm{BaSO}_{4}$ in the $\mathrm{B} 6$ tablet causes it to sink in the pylorus, as seen on the radiographs. The tablet went somewhat downwards after 2 hours, but it remained sinking in the pylorus until stomach emptying was complete. For the first 3 hours, the barium sulfate-labeled B6 tablet appears to be floating in the same position in the stomach. This could be due to the EC content's low density.

\section{Conclusion}

Promising pulsatile single pulse and floating double pulse core-in-cup tablets of metoprolol were successfully formulated. They provided a desirable lag time followed by rapid and complete drug release. This pulsatile drug delivery mechanism is clearly designed to release drugs in a chronological manner. For example, the sympathetic nervous system, heart rate variability, and the rapid rise in blood pressure have all been linked to the early morning hours. In this instance, pulsatile drug delivery techniques may be preferable. As a result, the medicine will only release when it is needed.

\section{REFERENCE}

1. Youan, B. B. C. (2010). Chronopharmaceutical drug delivery systems: Hurdles, hype or hope?. Advanced drug delivery reviews, 62(9-10), 898-903.

2. Sewlall, S., Pillay, V., P Danckwerts, M., E Choonara, Y., MK Ndesendo, V., \& C du Toit, L. (2010). A timely review of state-of-the-art chronopharmaceuticals synchronized with biological rhythms. Current drug delivery, 7(5), 370-388.

3. Sokar, M. S., Hanafy, A. S., El-Kamel, A. H., \& El-Gamal, S. S. (2013). Pulsatile core-in-cup valsartan tablet formulations: in vitro evaluation. asian journal of pharmaceutical sciences, 8(4), 234-243.

4. Kumar, J. (2013). Epidemiology of hypertension. Clinical Queries: Nephrology, 2(2), 56-61.

5. Messerli, F. H., Bangalore, S., \& Julius, S. (2008). Risk/benefit assessment of $\beta$-blockers and diuretics precludes their use for first-line therapy in hypertension. Circulation, 117(20), 27062715.

6. National Center for Biotechnology Information (2021). PubChem Compound Summary for CID 4171, Metoprolol. Retrieved September 2021 from https://pubchem.ncbi.nlm.nih.gov/compound/Metoprolol

7. Chaurasia, G. (2016). A review on pharmaceutical preformulation studies in formulation and development of new drug molecules. International Journal of Pharmaceutical Sciences and Research, 7(6), 2313. 
8. Train, D. (1958). Some aspects of the property of angle of repose of powders. Journal of Pharmacy and Pharmacology, 10(Supplement_1), 127T-135T.

9. Kishanrao, C. G., Harsha, S. S., Kumar, D. Y., Neelima, K. S. S. N., \& Sambamoorthy, U. (2013). Formulation and evaluation of colon specific matrix tablets of esomeprazole. Int J Current Trends Pharm Res, 1(4), 217-24.

10. Chavda, V. P., SONIWALA, V. R. M. M., \& CHAVDA, J. R. (2013). Preparation and Evaluation of Extended Release Nimesulide Tablet Based on Diffusion Controlled Mechanism. Asian Journal of Pharmaceutical Research and Health Care, 5(2).

11. Ramu, B. (2017). FORMULATION AND EVALUATION OF GASTRORETENTIVE FLOATING BIOADHESIVE TABLETS OF HYDROCHLORTHIAZIDE. Asian Journal of Pharmaceutical and Clinical Research, 150-155.

12. Divya, S., \& Ganesh, G. N. K. (2019). Characterization of Powder Flowability Using FT4Powder Rheometer. Journal of Pharmaceutical Sciences and Research, 11(1), 25-29.

13. Faix, O. (1992). Fourier transform infrared spectroscopy. In Methods in lignin chemistry (pp. 83-109). Springer, Berlin, Heidelberg. 